\title{
Secondary central nervous system lymphoma: spectrum of morphological MRI appearances
}

This article was published in the following Dove Press journal:

Neuropsychiatric Disease and Treatment

\author{
Hana Malikova ${ }^{1,2}$ \\ Miroslava Burghardtova' \\ Eva Koubska ${ }^{3}$ \\ Vaclav Mandys ${ }^{4}$ \\ Tomas Kozak ${ }^{5}$ \\ Jiri Weichet ${ }^{\prime}$
}

'Radiology Department, Third Faculty of Medicine, Faculty Hospital Kralovske Vinohrady, Charles University, Prague, Czech Republic; ${ }^{2}$ Institute of Anatomy, Second Faculty of Medicine, Charles University, Prague, Czech Republic; ${ }^{3}$ Radiology Department, Na Homolce Hospital, Prague, Czech Republic; ${ }^{4}$ Pathology Department, Third Faculty of Medicine, Faculty Hospital Kralovske Vinohrady, Charles University, Prague, Czech Republic; ${ }^{5} \mathrm{Hematooncology}$ Department, Third Faculty of Medicine, Faculty Hospital Kralovske Vinohrady, Charles University, Prague, Czech Republic
Correspondence: Jiri Weichet Third Faculty of Medicine, Faculty Hospital Kralovske Vinohrady, Charles University, Srobarova II50/50, Prague 10, CZ-I00 34, Czech Republic $\mathrm{Fax}+420267126409$ Email jiri.weichet@fnkv.cz
Background: Secondary central nervous system lymphoma (SCNSL) is a rare and aggressive disease, which is defined as secondary central nervous system (CNS) involvement in patients with systemic lymphoma. According to previous reports, SCNSL presents mostly with leptomeningeal spread; however, our experience differs. In the present study, we demonstrate the diversity of magnetic resonance imaging (MRI) patterns in SCNSL.

Patients and methods: Initial morphological MRI findings in 21 patients (10 women and 11 men with mean age $62.3 \pm 16.2$ years) with SCNSL were retrospectively evaluated. All patients suffered from neurological symptoms and underwent MRI, and all cases were histologically verified. Twelve patients were treated by corticosteroids at the time of the initial MRI.

Results: Parenchymal lesions were present in 18 of 21 cases $(85.7 \%)$, solitary meningeal infiltration was present in 1 patient $(4.8 \%)$, leptomeningeal infiltration in combination with hypophyseal involvement in 1 patient (4.8\%), and solitary involvement of the sixth cranial nerve $(\mathrm{CN})$ was found in 1 patient (4.8\%). Multiple lesions were present in 11 of 21 cases $(52.4 \%)$. Diffusion restriction in all or part of the lesion was detected in 14 of 18 cases $(77.8 \%)$. All parenchymal lesions had an infiltrative appearance and most enhanced homogenously (11 of 17 cases; 64.7\%). A combination of parenchymal and meningeal involvement was found in 10 of 21 cases (47.6\%). Infiltration of the $\mathrm{CNs}$, basal ganglia, corpus callosum, and ependyma was present in 8 of 21 cases (38.1\%) for each of the abovementioned structures; hypothalamichypophyseal axis was affected in 7 of 21 cases (33.3\%).

Conclusion: In contrast to previous reports, SCNSL presented as parenchymal disease. MRI is not sufficient for differentiation between primary and secondary CNS lymphoma.

Keywords: brain, parenchymal lesions, magnetic resonance, tumor

\section{Introduction}

Central nervous system lymphoma (CNSL) is an aggressive and rare brain neoplasm that may involve the brain, meninges, spinal cord, and eyes. CNSL is divided into 2 subtypes, primary central nervous system lymphoma (PCNSL) and secondary central nervous system lymphoma (SCNSL). In PCNSL, the central nervous system (CNS) is primarily involved without systemic lymphoma presence. SCNSL is defined as secondary CNS involvement in patients with systemic lymphoma; this type has also been referred to as metastatic lymphoma. SCNSL is an infrequent but nearly always fatal complication of systemic lymphoma occurring in $<1 \%$ of indolent systemic lymphomas and $<5 \%$ of aggressive systemic lymphomas when CNS prophylaxis is given. ${ }^{1,2}$ However, SCNSL may occur in up to $50 \%$ of cases of Burkitt/lymphoblastic lymphoma or AIDS-related lymphoma when no prophylaxis is given. ${ }^{3,4}$ Histologically, SCNSL is typically diffuse large B-cell lymphoma (DLBCL), while other types such as Burkitt lymphoma or T-cell lymphoma (T-cell non-Hodgkin lymphoma [T-NHL]) 
are less common and Hodgkin lymphoma is extremely rare., Lymphoma cells may reach the CNS by hematogenous spread, direct invasion from contiguous structures, or extension along nerves. ${ }^{7}$

According to literature data, approximately two-thirds of SCNSL cases present with leptomeningeal spread while one-third presents with brain parenchymal disease. ${ }^{7}$ Villa et al reported leptomeningeal infiltration in 29\% (9 of 31 ) of SCNSL cases, parenchymal involvement in 48\% (15 of 31 ) cases, and combined leptomeningeal and parenchymal involvement in $22 \%$ ( 7 of 31 ) cases. $^{4}$ In contrast to preexisting literature data, we observed SCNSL involvement differently at our center. Thus, the present study was performed to demonstrate the diversity of magnetic resonance imaging (MRI) patterns in our SCNSL patients.

\section{Patients and methods}

\section{Patient selection}

We retrospectively searched the hospital databases of both institutions (Faculty Hospital Kralovske Vinohrady and $\mathrm{Na}$ Homolce Hospital) for patients with histologically proven SCNSL, covering the time period of 2007-2016. We excluded all cases with per continuitatem spread of extracranial lymphoma intracranially. All patients underwent MRI examinations during the diagnostic process and images obtained at the time of initial neurological manifestation were evaluated.

\section{MRI examination}

All patients were examined on different whole-body MRI scanners, namely, GE Signa HDxT 1.5 T (GE Healthcare UK Ltd, Little Chalfont, UK), Siemens Magnetom Avanto 1.5 T (Siemens AG, Munich, Germany), Siemens Magnetom Symphony 1.5 T (Siemens AG), and Siemens Magnetom Skyra 3 T (Siemens AG). The MRI protocols included the following sequences: T2-weighted fast spin echo in axial and/or coronal planes, gradient echo $\mathrm{T} 2 *$ in the axial plane, diffusion-weighted imaging $(b=0, b=1,000)$ in the axial plane, T2-weighted fluid-attenuated inversion recovery in the axial plane, and T1-weighted spin echo in the sagittal and/or coronal planes. In 20 patients, gadolinium-based contrast agents were administrated intravenously; post-contrast sequences included T1-weighted spin echo in different planes and T1-weighted 3D gradient echo.

The following signs were assessed on MRI images:

- lesion localization: supratentorial, infratentorial, both;

- quantity of lesions: solitary, multiple, diffuse infiltrative;

- quality of lesions: demarcated, infiltrative;
- extent and type of parenchymal lesion enhancement: predominantly homogeneous enhancement $(>70 \%$ of lesion); inhomogeneous enhancement $(<70 \%,>30 \%$ of lesion); predominately non-enhancing ( $<30 \%$ of lesion); worm-like, strip, patchy, or misty enhancement;

- involvement of basal ganglia, cranial nerves (CNs), corpus callosum, meninges, ependyma, and structures of hypothalamic-hypophyseal axis;

- presence of diffusion restriction; signs of hemorrhage; edema.

Definitions of all lesion/infiltration types and inclusion criteria used in the MRI assessment are summarized in Table 1.

All MRI data were independently analyzed by 3 experienced radiologists who raised uniform MRI reports.

\section{Ethics approval and consent to participate}

All patients included in the study provided written informed consent to undergo treatment including diagnostic procedures (including MRI) and agreed to the publication of medical data and images in an anonymous form. The informed consent forms are maintained on files. This retrospective study conforms to the Declaration of Helsinki and was approved by the ethics committee of the Faculty Hospital Kralovske

Table I Definitions of all types of lesions and infiltrations

\begin{tabular}{ll}
\hline Type of lesion & Definition/inclusion criteria \\
\hline Solitary lesion & $\begin{array}{l}\text { A single lesion in either brain parenchyma, } \\
\text { focal meningeal, or ependymal localization, } \\
\text { or single cranial nerve involvement. The } \\
\text { continuous spread through several neuronal } \\
\text { structures was considered as a solitary lesion. } \\
\text { A combination of } 2 \text { or more solitary lesions } \\
\text { mentioned above. } \\
\text { Extensive continuous infiltration of both } \\
\text { white and gray matter by non-enhancing } \\
\text { and/or enhancing tumorous involvement } \\
\text { (from homogeneous to patchy, worm- } \\
\text { like, stripy, misty, etc), which spread along } \\
\text { large white matter tracts and continuously } \\
\text { affected at least a) }>2 / 3 \text { of I cerebral } \\
\text { hemisphere and/or b) different extents of } \\
\text { both supratentorial and infratentorial regions } \\
\text { and/or different extents of both hemispheres. } \\
\text { involvement }\end{array} \quad \begin{array}{l}\text { Thickening and enhancing meningeal/ } \\
\text { ependymal surfaces, which can be smooth, } \\
\text { irregular, or nodular. } \\
\text { Thickening of cranial nerves and their T2 } \\
\text { hyperintense and/or enhancing portions, } \\
\text { which can be smooth, irregular, or nodular. }\end{array}$ \\
\hline
\end{tabular}


Vinohrady (number 2017/11) and the Na Homolce Hospital (number 2016/06), Prague, Czech Republic. Trial registration was not required.

\section{Statistics}

Data were expressed as mean $\pm \mathrm{SD}$. Categorical values were analyzed using the chi-squared test. $P$-values $<0.05$ were considered significant. Analyses were performed using STATISTICA software version 12 (Tibco Software Inc, Palo Alto, CA, USA).

\section{Results}

\section{Patients and clinical data}

Twenty-one patients with verified SCNSL were included; 10 women, 11 men, mean age $62.3 \pm 16.2$ years (range $23-83$ years). Ten (47.6\%) cases were histologically proven by stereotactic biopsy, 6 (28.6\%) cases by autopsy, 3 (14.3\%) cases by open neurosurgery, and $2(9.5 \%)$ cases were verified by flow cytometry of the cerebrospinal fluid. Nineteen patients had a history of known and treated systemic lymphoma. In 2 cases, brain involvement was the first manifestation of systemic disease. Nineteen cases were diagnosed as B-cell non-Hodgkin lymphoma including 13 cases of DLBCL; T-NHL was confirmed in 2 cases.

The patients presented with various clinical manifestations (in most patients a combination of several symptoms). The most common clinical presentations were organic brain syndrome and headache in $10(47.6 \%)$ patients; the second most common manifestation was phatic disorder in $7(33.3 \%)$ patients. Some types of paresis and dysesthesia or paresthesia were reported in $5(23.8 \%)$ patients, and visual and/or hearing disturbances were found in $4(19 \%)$ patients. Other presentations such as cerebellar syndrome or seizure were not common and presented in 3 (14.3\%) and 2 (9.5\%) cases, respectively.

The duration between neurological symptoms onset and MRI examination was $12 \pm 15$ days (range $1-60$ days). Twelve $(57.1 \%)$ patients had used glucocorticoids at the time of initial MRI.

\section{MRI findings}

Seventeen patients underwent the complete diagnostic protocol; in 1 case gadolinium contrast agent was not administrated, and in 3 cases diffusion-weighted imaging was not performed.

Parenchymal involvement was present in 18 of 21 patients (85.7\%), multiple lesions in 11 of 21 cases $(52.4 \%)$. The lesions were localized mostly supratentorially in 12 of
21 cases $(57.1 \%)$. Restricted diffusion was observed in 14 of 18 cases $(77.8 \%)$. All parenchymal lesions had an infiltrative appearance, and most demonstrated homogeneous enhancement (11 of 17 cases; $64.7 \%$ ). In 10 of 21 cases (47.6\%), a combination of parenchymal and meningeal involvement was found. Infiltration of the $\mathrm{CNs}$, basal ganglia, corpus callosum, and ependyma was detected relatively often, each presented in 8 of 21 cases (38.1\%). Solitary infiltration of the sixth $\mathrm{CN}$ was present in 1 case, other cases involved the parenchyma and several CNs. CN5, CN7, and CN8 were affected 3 times and CN3 twice. Structures of the hypothalamic-hypophyseal axis were affected in 7 of 21 cases (33.3\%). Signs of intralesional hemorrhage were present only in 1 case, which is presented as a case report (see "Case 1"). Detailed diagnostic MRI results of the entire patient group are summarized in Table 2.

The patients were further divided into 2 subgroups according to corticosteroid therapy at the time of the initial MRI examination; glucocorticoids users (12 subjects) and non-glucocorticoids users ( 9 subjects). We found significant difference between the 2 groups in CNs involvement, with exception of the optic nerves and tracts. In glucocorticoids users, CNs were infiltrated in 5 (41.7\%) subjects, while in non-glucocorticoids users no $\mathrm{CN}$ infiltration was detected $(P=0.045)$. No other significant differences in MRI findings were found.

The typical MRI appearance of SCNSL in our group of patients is shown in Figure 1. Hypothalamic-hypophyseal axis involvement in another subject is shown in Figure 2. Moreover, we selected 2 interesting tricky SCNSL cases that differed from the more common MRI findings in our patients.

\section{Case I}

A 74-year-old male suffered from pleomorphic clinical symptoms since 2013, when Evans syndrome was diagnosed (vitamin B12, lactic acid, and iron deficiency). Thereafter, he was regularly followed hematologically. Some unclear neurological symptoms were reported by his son in October 2016. A computed tomography (CT) scan of the brain showed a single minor postischemic lesion in the white matter in the left frontal lobe. In January 2017, his cognition and orientation skills suddenly deteriorated. He was admitted to the neurology ward on February 1 and underwent a brain CT scan the same day, where progression of the initial finding was recognized and new ischemic lesions in the brain were described. The following day an MRI examination of the brain was performed. Several lesions in the white matter were found; all lesions showed restricted diffusion and signs of hemorrhage. 
Table 2 MRI findings in SCNSL

\begin{tabular}{|c|c|c|c|}
\hline MRI findings & $\begin{array}{l}\text { Number of } \\
\text { cases (\%) }\end{array}$ & MRI findings & $\begin{array}{l}\text { Number of } \\
\text { cases (\%) }\end{array}$ \\
\hline Localization & & Cranial nerve involvement & \\
\hline Supratentorial only & 12 of $21(57.1 \%)$ & Optic nerves & 6 of $21(28.6 \%)$ \\
\hline Infratentorial only & I of 2 I (4.8\%) & Other nerves & 5 of $21(23.8 \%)$ \\
\hline Both & 8 of $21(38.1 \%)$ & All cases of cranial nerves involvement & 8 of $21(38.1 \%)$ \\
\hline Type of lesion & & Meningeal involvement & \\
\hline Solitary & 6 of $21(28.6 \%)$ & Solitary & I of 2 I (4.8\%) \\
\hline Multiple & II of 2 I $(52.4 \%)$ & Combination with parenchymal lesions & 10 of $21(47.6 \%)$ \\
\hline Diffuse infiltrative & 4 of $21(19.0 \%)$ & Combination with another lesion & I of 21 (4.8\%) \\
\hline \multicolumn{4}{|l|}{ Parenchymal lesion enhancement* } \\
\hline Homogenous $>70 \%$ of lesion & II of I7 (64.7\%) & Ependymal involvement & 8 of 21 (38.1\%) \\
\hline Non-homogenous between $30 \%$ and $70 \%$ of lesion & 3 of $17(17.6 \%)$ & Basal ganglia involvement & 8 of 21 (38.1\%) \\
\hline Non-homogenous $<30 \%$ of lesion & 0 of 17 & Diffusion restriction** & 14 of $18(77.8 \%)$ \\
\hline Worm-like, strip, patchy, misty & 2 of $17(11.8 \%)$ & Corpus callosum involvement & 8 of 21 (38.1\%) \\
\hline No enhancement & I of I 7 (5.8\%) & Signs of hemorrhage & I of 2 I (4.8\%) \\
\hline Hypothalamic-hypophyseal axis involvement & & Vasogenic edema & \\
\hline Hypothalamus only & 5 of $21(23.8 \%)$ & Visible & 13 of 21 (6I.9\%) \\
\hline Hypophysis only & I of 2 I (4.8\%) & None & 8 of $21(38.1 \%)$ \\
\hline Both & I of $2 \mid(4.8 \%)$ & & \\
\hline
\end{tabular}

Notes: *Parenchymal lesions were present in 18 patients; however, in I patient contrast was not administrated; thus, 17 patients were evaluated. **Presence of diffusion restriction was assessed in only 18 patients; diffusion-weighted imaging was not acquired in 3 patients.

Abbreviations: MRI, magnetic resonance imaging; SCNSL, secondary central nervous system lymphoma.
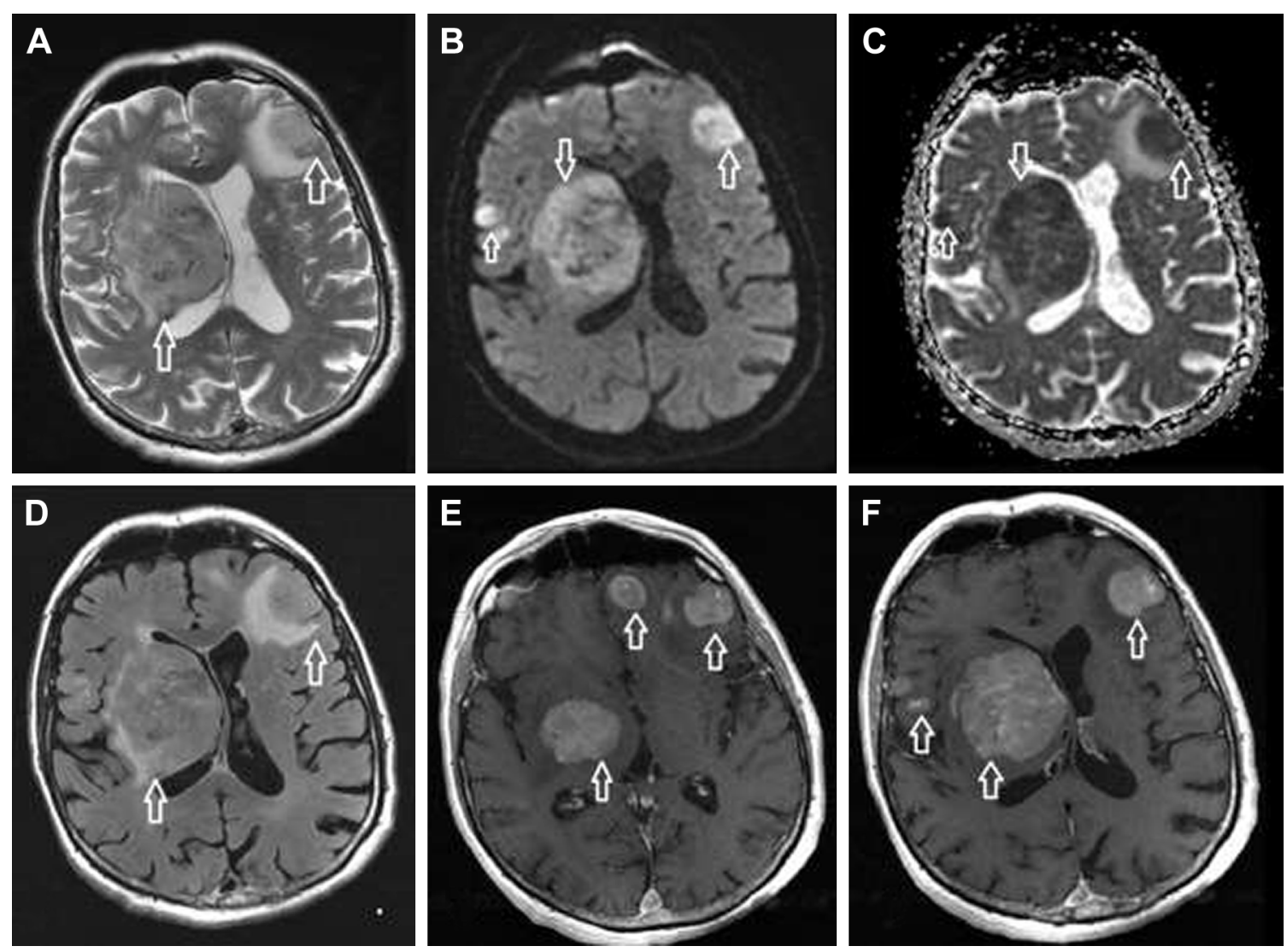

Figure I Common MRI appearance of SCNSL in our group.

Notes: Multiple lesions in brain parenchyma with intermediate intensity on T2-weighted images (A; arrows), with restricted diffusion on diffusion-weighted imaging (B; arrows) and ADC map (C; arrows). Visible vasogenic edema around the lesions on FLAIR (D; arrows). Strong enhancement is present after gadolinium injection on TI-weighted images (E and $\mathbf{F}$; arrows).

Abbreviations: ADC, apparent diffusion coefficient; FLAIR, fluid-attenuated inversion recovery; MRI, magnetic resonance imaging; SCNSL, secondary central nervous system lymphoma. 

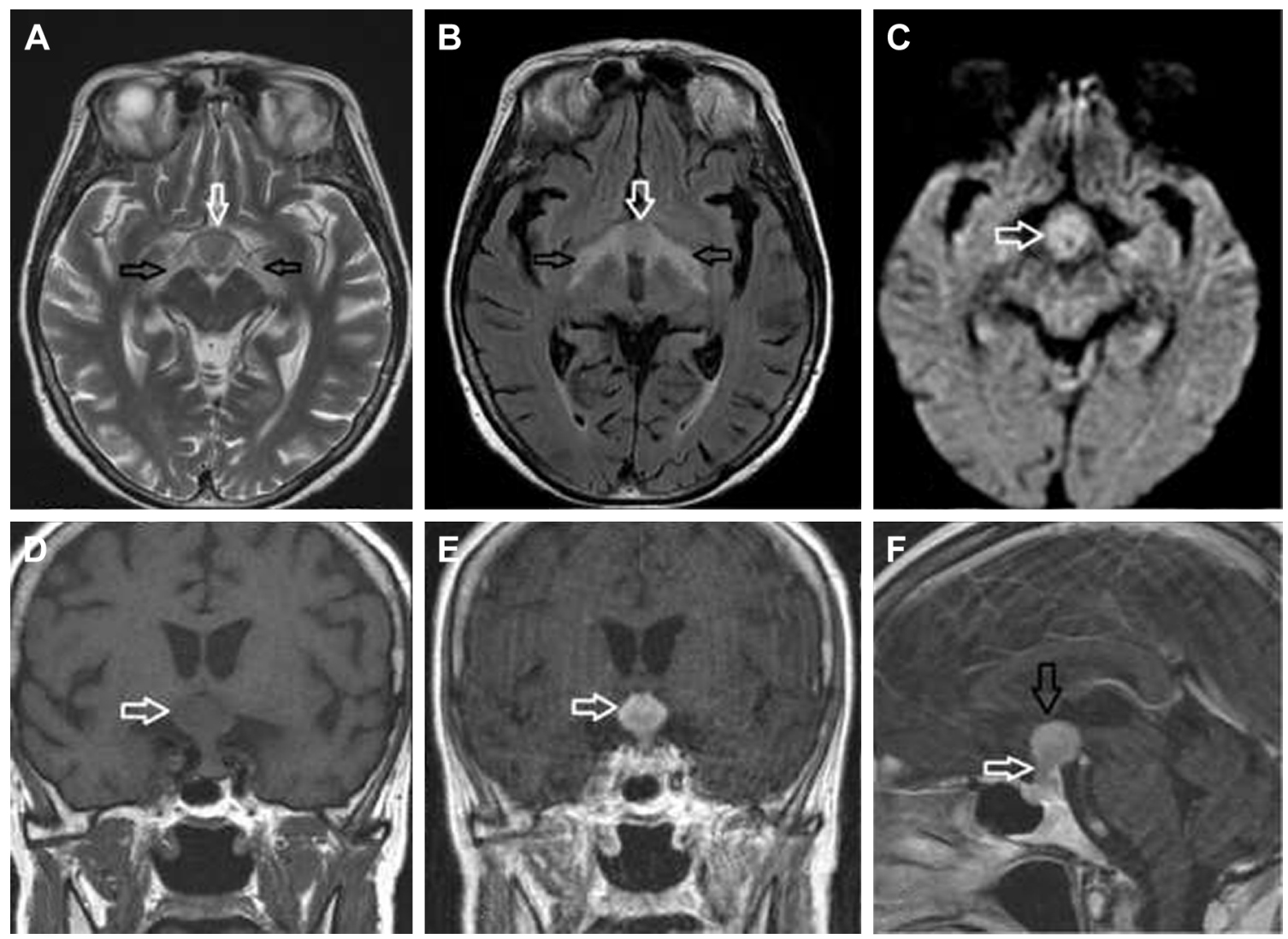

Figure 2 SCNSL with hypothalamic-hypophyseal axis involvement.

Notes: Hypothalamus (white arrow) and optic tracts (black arrows) involvement is visible on T2-weighted images (A) and FLAIR (B), with restricted diffusion in the hypothalamic lesion (C; arrow). The hypothalamic lesion was strongly enhanced after gadolinium injection compared to native TI-weighted images (D; arrow) and postcontrast TI-weighted images (E; arrow). Sagittal post-contrast TI-weighted images (F) show both hypothalamic (black arrow) and hypophyseal involvement with infundibular thickening (white arrow).

Abbreviations: FLAIR, fluid-attenuated inversion recovery; SCNSL, secondary central nervous system lymphoma.

A gadolinium-based contrast agent was not administrated. The lesions were described as multiple ischemic lesions (Figure 3). On February 7, his overall condition worsened and he was transferred to the intensive care unit; however, despite intensive care, he died within a few hours. An autopsy was performed and DLBCL was found in the retroperitoneum with left adrenal destruction and vascular dissemination to the lungs, brain, bone marrow, kidney, colon, and myocardium. Proven SCNSL was consistent with an intravascular angiocentric type of lymphoma. Unfortunately, proper diagnosis during the life of the patient was not established.

\section{Case 2}

A 74-year-old woman was diagnosed with peripheral T-cell lymphoma and treated in 2013. Relapse of the disease occurred in 2015. Six cycles of combined chemotherapy (rituximab, cyclophosphamide, doxorubicin, vincristine) as well as glucocorticoid therapy were completed in January 2016. From August 2016, the patient was considered to be in remission. Worsening sensomotoric polyneuropathy, new psychiatric disorders, as well as speech and memory impairments were observed in September 2016. The patient was admitted to the hospital and brain MRI was performed, where suspicion of CNS relapse was raised. Additionally, John Cunningham virus positivity was determined and the patient was diagnosed with progressive multifocal leukoencephalopathy. Lymphoma treatment was then ended and only symptomatic therapy was initiated. In January 2017, the patient began to complain about very painful skin lesions and was given corticosteroid therapy with very good effect on the brain lesion, as proven by MRI in April 2017. On the last MRI exam in August 2017, progression of the brain affection with new infiltrations in the brain stem and right half of the cerebellum was found. The MRI appearance at the 3 time points mentioned above is illustrated in Figure 4. Autopsy confirmed a diagnosis of SCNSL.

\section{Discussion}

In the present study, we have demonstrated variable SCNSL appearances at the time of initial diagnosis. We can clearly 

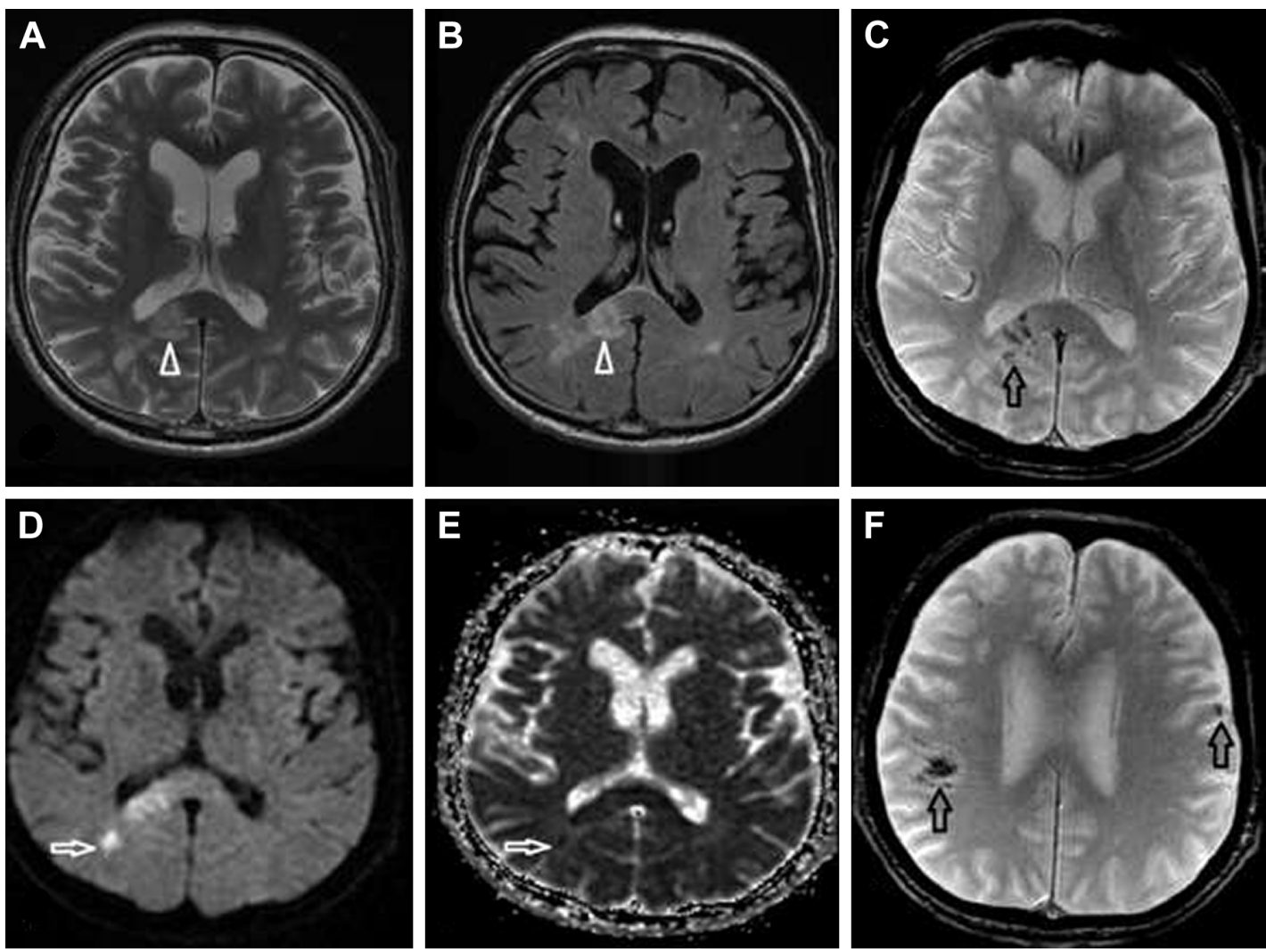

Figure 3 SCNSL mimicking multiple ischemic lesions.

Notes: Multiple hyperintense lesions on T2-weighted images (A) and FLAIR (B) were observed (white arrowheads). The lesions showed diffusion restriction (D; white arrow) and low signal on the ADC map (E; white arrow). Nearly all lesions had signs of hemorrhage on the gradient echo sequence (C and $\mathbf{F}$; black arrows). Abbreviations: ADC, apparent diffusion coefficient; FLAIR, fluid-attenuated inversion recovery; SCNSL, secondary central nervous system lymphoma.
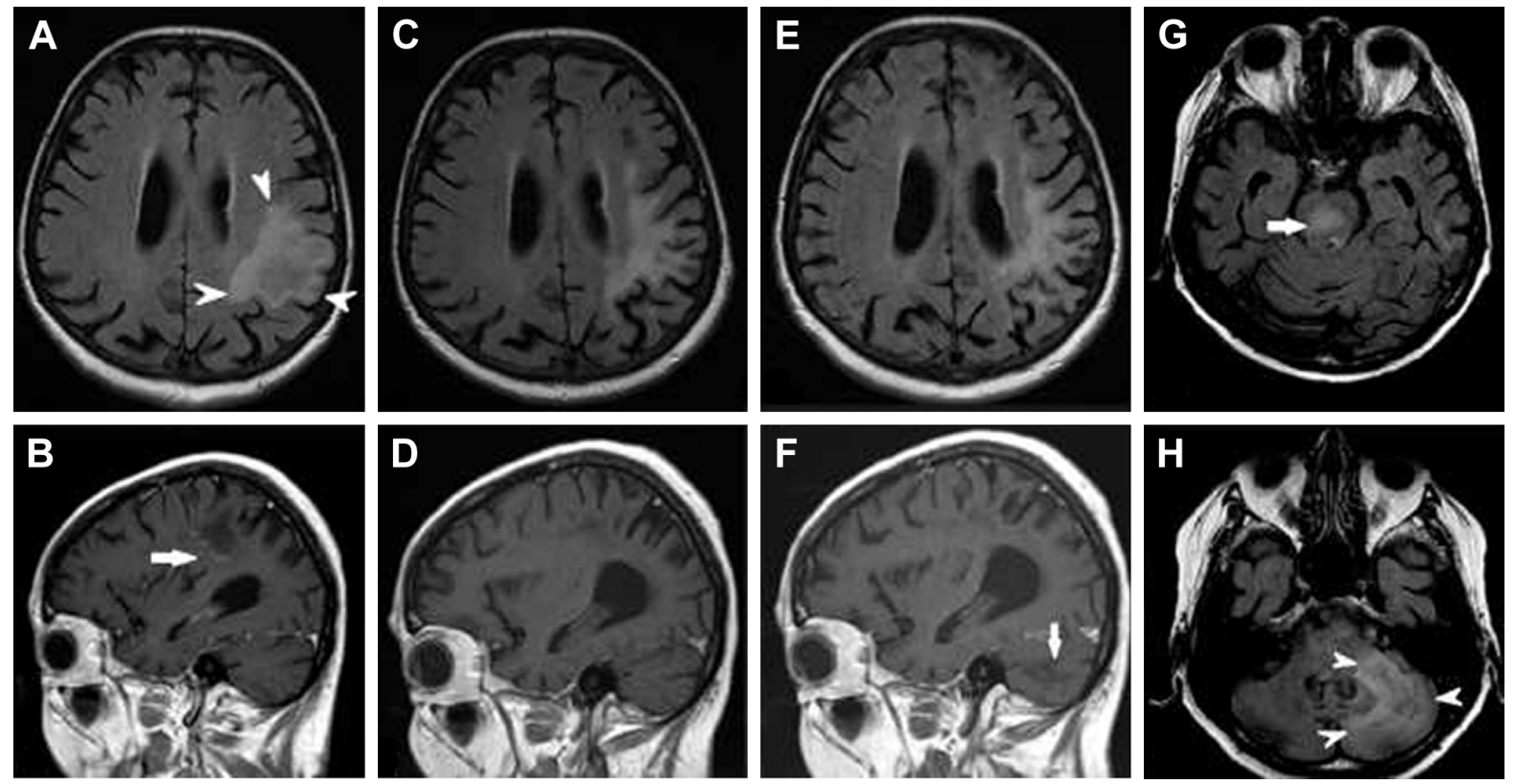

Figure 4 SCNSL mimicking PML.

Notes: Initial MRI at the time of new neurological symptoms showed a single hyperintense lesion on FLAIR (A; arrowheads) with subtle worm-like inhomogeneous enhancement after gadolinium injection on TI-weighted images (B; arrow). On follow-up MRI 7 months later (C and $\mathbf{D})$ the brain lesion decreased in size, and post-contrast enhancement on $\mathrm{TI}$-weighted images completely regressed. The final MRI 4 months later at the time of clinical relapse (E-H) demonstrated that the initial lesion had not changed (E); however, multiple new infiltrative lesions appeared hyperintense on FLAIR, primarily in the brain stem (G; arrow) and cerebellum (H; arrowheads), without post-contrast enhancement on TI-weighted images (F; arrow).

Abbreviations: FLAIR, fluid-attenuated inversion recovery; MRI, magnetic resonance imaging; PML, progressive multifocal leukoencephalopathy; SCNSL, secondary central nervous system lymphoma. 
state that, in our patients, in contrast to previous studies most of SCNSL affections were parenchymal lesions., ${ }^{4,8}$ Parenchymal lesions were present in $85.7 \%$ of patients. Solitary meningeal infiltration was very rare, present in only 1 case (4.8\%). Most of lesions were multiple and supratentorially localized, and restricted diffusion in all or some part of the lesions was often seen. All parenchymal lesions had an infiltrative appearance, and most of them enhanced homogeneously ( $64.7 \%$ of cases). Infiltration of $\mathrm{CNs}$, basal ganglia, corpus callosum, and ependyma was relatively frequent. As stated earlier, our findings differ from most previous reports. ${ }^{7}$ The findings of 14 previous studies from 1985 to 2004 were summarized in a review by Hill and Owen. ${ }^{8}$ They reported meningeal involvement in $66 \%$ of patients with SCNSL but brain involvement in only $33 \%$ of patients. However, in most of the studies reviewed, the findings were not based on a combination of MRI examinations and histological verification and thus the diagnosis was not unequivocally proven. In some studies, the diagnosis was made by cerebrospinal fluid examination, by $\mathrm{CT}$, or only from clinical data without histological or radiological validation. ${ }^{9-12}$ In a study published by Villa et al, the authors reported nearly onethird of leptomeningeal involvement, nearly $50 \%$ of cases had parenchymal lesions, and the remainder showed both leptomeningeal and parenchymal involvement in SCNSL. ${ }^{4}$ However, only $55 \%$ of their subjects underwent MRI or CT; in the reminder, the diagnosis was made using cerebrospinal fluid flow cytometry or only from clinical data, without histological or imaging confirmation. All of our patients suffered from neurological symptoms. Nearly all (91\%) MRI findings were histologically proven by stereotactic biopsy, open surgery, or autopsy. In only 2 of 21 cases, cerebrospinal flow cytometry was performed to confirm the diagnosis of SCNSL. According to Pittman et al, this diagnostic tool has both high positive and negative predictive values in patients with a history of lymphoma and/or abnormal brain imaging, when compared with open brain tissue biopsy. ${ }^{13}$ Thus, we consider our data valid.

CNSL variably changes over a relatively short period of time and the clinical manifestation and MRI appearances may be dramatically affected by corticosteroid administration. The temporary regression of CNSL during corticosteroid therapy, which may be partial or even complete, is well known. ${ }^{14,15}$ However, in a minority of cases, progression after corticosteroid therapy may be observed..$^{15}$ Moreover, spontaneous regression of CNSL has been repeatedly described, mostly in solitary cases. ${ }^{16}$ In a study by Koubska et al, statistically significant differences in morphological MRI findings between immunocompromised and immunocompetent patients with CNSL were reported. ${ }^{15}$ In that study, all immunocompromised patients with non-homogenously enhancing lesions were treated with corticosteroids. The authors speculated that the enhancement differences between immunocompromised and immunocompetent patients may be related to corticosteroid therapy. ${ }^{15}$ In our study, nearly $60 \%$ of patients were treated by glucocorticoids at the time of initial MRI. We found just 1 significant difference between glucocorticoid users and non-users in the involvement of $\mathrm{CNs}$, aside from the optic nerves. All CNs infiltrations were present in the glucocorticoid user subgroup. We do not have any explanation for this finding. It may be related only to the small sample size. Despite the aforementioned observations, the morphological MRI findings of the entire patient group were very similar to the previously published findings in PCNSL. ${ }^{17,18}$ Our study supports the results of a few earlier studies, which did not find significant differences in MRI appearances between PCNSL and SCNSL. ${ }^{15,19}$

The present study has several limitations. The cohort of patients was small, as SCNSL is a rare disease, and we included histologically verified cases only. Due to its retrospective nature, some MRI examinations were of lower quality and acquired on different whole-body MRI scanners. We did not have sufficient data for reviewing advanced MRI techniques such as magnetic resonance spectroscopy, perfusion imaging, or diffusion tensor imaging. Not all patients underwent diffusion-weighted imaging.

\section{Conclusion}

In contrast to previously published reports, SCNSL presented primarily as parenchymal disease in our patients, and its MRI appearance did not differ from the morphological MRI findings in PCNSL described previously. Post-contrast MRI is an important diagnostic tool, but insufficient to make a clear diagnosis or discriminate PCNSL from SCNSL. Histological verification of brain lesions is still mandatory, although stereotactic biopsy is not without complications. ${ }^{20}$ Systemic lymphoma must be excluded in the case of all histologically proven brain lymphoma lesions, as neither MRI nor histology itself is able to distinguish between PCNSL and SCNSL. In some cases, MRI appearance of lymphoma is tricky as we have demonstrated separately. A multidisciplinary approach for the next research is recommended.

\section{Availability of data and materials}

Data and materials are presented in the main body of the paper and in additional supporting files (tables). The raw MRI data are stored in digital form at at the Radiology Department, Faculty Hospital Kralovske Vinohrady, Prague, Czech 
Republic, and at the Radiology Department, Na Homolce Hospital, Prague, Czech Republic.

\section{Acknowledgment}

This work was supported by the Charles University research program PROGRES Q 28 (Oncology).

\section{Author contributions}

All authors contributed toward data analysis, drafting and critically revising the paper, gave final approval of the version to be published, and agree to be accountable for all aspects of the work.

\section{Disclosure}

The authors report no conflicts of interest in this work.

\section{References}

1. Haioun C, Besson C, Lepage E, et al. Incidence and risk factors of central nervous system relapse in histologically aggressive non-Hodgkin's lymphoma uniformly treated and receiving intrathecal central nervous system prophylaxis: a GELA study on 974 patients. Groupe d'Etudes des Lymphomes de l'Adulte. Ann Oncol. 2000;11(6):685-690.

2. Jahnke K, Thiel E, Martus P, Schwartz S, Korfel A. Retrospective study of prognostic factors in non-Hodgkin lymphoma secondarily involving the central nervous system. Ann Hematol. 2006;85(1):45-50.

3. Bernstein SH, Unger JM, Leblanc M, Friedberg J, Miller TP, Fisher RI. Natural history of CNS relapse in patients with aggressive non-Hodgkin's lymphoma: a 20-year follow-up analysis of SWOG 8516 - the Southwest Oncology Group. J Clin Oncol. 2009;27(1):114-119.

4. Villa D, Connors JM, Shenkier TN, Gascoyne RD, Sehn LH, Savage KJ. Incidence and risk factors for central nervous system relapse in patients with diffuse large B-cell lymphoma: the impact of the addition of rituximab to CHOP chemotherapy. Ann Oncol. 2010;21(5):1046-1052.

5. Bierman P, Giglio P. Diagnosis and treatment of central nervous system involvement in non-Hodgkin's lymphoma. Hematol Oncol Clin North Am. 2005;19(4):597-609.
6. Grimm S, Chamberlain M. Hodgkin's lymphoma: a review of neurologic complications. Adv Hematol. 2011;2011:624578.

7. Haldorsen IS, Espeland A, Larsson E-M. Central nervous system lymphoma: characteristic findings on traditional and advanced imaging. Am J Neuroradiol. 2011;32(6):984-992.

8. Hill QA, Owen RG. CNS prophylaxis in lymphoma: who to target and what therapy to use. Blood Rev. 2006;20(6):319-332.

9. Keldsen N, Michalski W, Bentzen SM, Hansen KB, Thorling K. Risk factor for central nervous system involvement in non-Hodgkinslymphoma - a multivariable analysis. Acta Oncol. 1996;35(6):703-708.

10. van Besien K, Ha CS, Murphy S, et al. Risk factors, treatment, and outcome of central nervous system recurrence in adults with intermediategrade and immunoblastic lymphoma. Blood. 1998;91(4):1178-1184.

11. Zinzani PL, Magagnoli M, Ferezza G, et al. Isolated central nervous system relapse in aggressive non-Hodgkin's lymphoma: the Bologna experience. Leuk Lymphoma. 1999;32(5-6):571-576.

12. Hollender A, Kvaloy S, Nome O, Skovlund E, Lote K, Holte H. Central nervous system involvement following diagnosis of non-Hodgkin's lymphoma: a risk model. Ann Oncol. 2002;13(7):1099-1107.

13. Pittman M, Treese S, Chen L, et al. Utility of flow cytometry of cerebrospinal fluid as a screening tool in the diagnosis of central nervous system lymphoma. Arch Pathol Lab Med. 2013;137(11): $1610-1618$

14. Bhagavathi S, Wilson JD. Primary central nervous system lymphoma. Arch Pathol Lab Med. 2008;132(11):1830-1834.

15. Koubska E, Weichet J, Malikova H. Central nervous system lymphoma: a morphological MRI study. Neuro Endocrinol Lett. 2016;37(4): 318-324.

16. Hernández Rubio L, Giner Bernabeu JC, Perez Sempere A, Toro P. Primary cerebral lymphoma with spontaneous remission. Neurologia. 2013;28(2):123-126.

17. Malikova H, Koubska E, Weichet J, et al. Can morphological MRI differentiate between primary central nervous system lymphoma and glioblastoma? Cancer Imaging. 2016;16(1):40.

18. Haldorsen IS, Kråkenes J, Krossness BK, Mella O, Espeland A. CT and MR imaging features of primary central nervous system lymphoma in Norway, 1989-2003. Am J Neuroradiol. 2009;30(4):744-751.

19. Senocak E, Oguz KK, Ozgen B, et al. Parenchymal lymphoma of the brain on initial MR imaging: a comparative study between primary and secondary brain lymphoma. Eur J Radiol. 2011;79(2):288-294.

20. Malikova H, Liscak R, Latnerova I, et al. Complications of MRIguided stereotactic biopsy of brain lymphoma. Neuro Endocrinol Lett. 2014;35(7):613-618.
Neuropsychiatric Disease and Treatment

\section{Publish your work in this journal}

Neuropsychiatric Disease and Treatment is an international, peerreviewed journal of clinical therapeutics and pharmacology focusing on concise rapid reporting of clinical or pre-clinical studies on a range of neuropsychiatric and neurological disorders. This journal is indexed on PubMed Central, the 'PsycINFO' database and CAS,

\section{Dovepress}

and is the official journal of The International Neuropsychiatric Association (INA). The manuscript management system is completely online and includes a very quick and fair peer-review system, which is all easy to use. Visit http://www.dovepress.com/testimonials.php to read real quotes from published authors. 